\title{
Evaluate comparative efficacy of tuberculin test, gross and histopathology, Ziehl-Neelsen staining and polymerase chain reaction technique for the detection of tuberculosis in dairy cattle
}

\author{
Md. Shamim Al Mamun, Tahmina Ruba, Md. Zakir Hossain, Munmun Pervin \\ and Mohammad Abu Hadi Noor Ali Khan* \\ (Department of Pathology, Faculty of Veterinary Science, Bangladesh Agricultural University, Mymensingh- \\ 2202, Bangladesh*
}

\begin{abstract}
Tuberculosis (TB) is an archaic infectious disease of human and animals caused by Mycobacterium spp and responsible for considerable morbidity and mortality. TB in man and animals is mostly caused by Mycobacterium bovis (M. bovis, bovine TB) and Mycobacterium tuberculosis (M. tuberculosis, human TB). This study, was, aimed at rapid and specific identification of bovine $T B$ and human $T B$ in dairy cattle by using intradermal tuberculin test, gross and microscopic pathology, Ziehl-Neelson staining of tissue sections and PCR detection of the specific causes of TB. Out of 100 dairy cattle tested using intradermal tuberculin test in the Bangladesh Agricultural University dairy farm during the period between 2013 and 2014, 05 yielded hypersensitivity reactions to intradermal tuberculin test. The tuberculin test positive cattle $(N=5)$ were sacrificed and investigated systematically. Gross lesions observed were recorded during necropsy and lungs, liver, spleen, kidney and lymphnodes were collected in 10\% buffered neutral formalin for histopathological examination. A small portion of lungs were snap frozen and preserved at $-20^{\circ} \mathrm{C}$ for the extraction of genomic DNA and PCR detection of TB. Grossly nodular masses in lungs and enlarged mesenteric lymphnodes were observed in four cases. Accumulation of macrophages, lymphocytes and fibrous connective tissue was seen in microscopic section of lungs, spleen and lymphnodes. Tissue sections stained with Ziehl Neelsen staining, showed rod shaped bright red color acid fast bacilli in three cattle. The multiplex PCR used successfully amplified fragment of 16srRNA gene specific for Mycobacterial infections (1030bp) and infection due to Mycobacterium tuberculosis complex (372bp) in four cases. Results of uniplex PCR showed that three cattle were infected with M. bovis (MPB83 gene specific 600bp amplicon) and one cattle with M. tuberculosis (H37Rv Rv3479HP gene specific 667bp amplicon). Out of five tuberculin test positive cattle sacrificed in this study, four were found to infect with TB; one cattle could have reacted due to infection with saprophytic mycobacterium or other infections that cross reacted to tuberculin test. The multiplex and uniplex PCR adapted and designed in this study found sensitive, accurate and conclusive. Dairy cattle in this farm were infected with M. bovis and M. tuberculosis. M. bovis and M. tuberculosis are extremely zoonotic pathogens. It needs to examine all of the dairy cattle with tuberculin test and PCR test twice in a year, sacrifice the test positive cattle at early onset to prevent dissemination in farm animals and human. People working in the farms also need to sit for tuberculin test, Ziehl Neelsen staining and PCR test of cough to detect infected and carrier state of infection.
\end{abstract}

Keywords: Tuberculosis, dairy cattle, diagnosis, PCR, pathology

\section{Introduction}

Bangladesh is an agrarian country and density of human population is intermingling with their livestock. There are about 24.2 million cattle, 1.3 million buffaloes, 21.6 million goats and 2.2 million sheep population in Bangladesh [1]. Livestock and human being are, therefore, shared common habitation as well as a number of infectious diseases. In Bangladesh, Tuberculosis (TB) is a major bacterial disease that hinders the livestock production. In general, TB in farms impose strict restriction on international trade due to a major zoonotic threat [2],[3]. TB due to M. tuberculosis mostly affect lungs, leading morbidity and mortality due to respiratory failure [19].

Tuberculosis (TB) is one of the most widespread infectious diseases, caused mainly by $M$. tuberculosis and one of the leading cause of human and animal death. Bovine tuberculosis (BTB) is widely zoonotic, caused by infection with the slow-growing, bacterium $M$. bovis and $M$. tuberculosis [4]. The animal were human TB, caused by $M$. bovis leading extra-pulmonary infection and other organ/systems lymph nodes, skin, skeletal, genito-urinary and central nervous system (meningitis) [5]. TB due to M. tuberculosis mostly affect lungs, leading morbidity and mortality due to respiratory failure [3].

The diagnosis of Mycobacterium-induced disease is most commonly made by tuberculin test Xray and pathological investigation. Acid-fast bacilli (AFB) detection has low sensitivity and specificity, and, albeit to, can only provide a preliminary diagnosis. However, long time is necessary for M. bovis and M. tuberculosis to 
Evaluate comparative efficacy of tuberculin test, gross and histopathology, Ziehl-Neelsen staining..

grow in culture. Even though, non-fastidious microorganisms contaminate a small percentage of cultures [6]. Currently, polymerase chain reaction (PCR) is the most promising technique for approaching this diagnostic dilemma which has been used to amplify different regions of the Mycobacterial genome as well as species identification in a variety of specimens. Although, the rapid and accurate detection of Mycobacterium spp. have profound significance in effective treatment, control and eradication of TB in man and animals. The deletion technique is not much efficient and available as well. The present study was aimed at specific detection of tuberculosis at Bangladesh Agricultural University (BAU) Dairy Farm. The dairy cattle (N=250) of BAU is often used to demonstrate practical classes for the students of both Veterinary Science and Animal husbandry and also there is free access of farm workers and public. It was, therefore, necessary to test dairy cattle to know the level of infectivity due to TB by using tuberculin test, gross and histopathology and PCR and dispose the test positive animals to prevent future zoonosis.

\subsection{Sample collection}

\section{Materials And Methods}

The investigation of tuberculosis (TB) was carried out in dairy cattle of Bangladesh Agricultural University Dairy Farm; a total of 100 dairy cattle were tested using intradermal tuberculin test. The Single Intradermal Tuberculin test (SITT) was performed onto the caudal fold of skin [7]. About 0.1ml of Bovine PPD was injected within the skin of caudal tail fold by using a short needled syringe (McLintock ${ }^{\circledR}$ preset syringe and tuberculin testing equipment, Glasgow, G81 1NH). The immune response to the PPD was recognized by observing the swelling or discoloration at the site where the PPD was injected. If any abnormalities such as discoloration or swelling were noted at the injection site, then the animal was regarded as tuberculin test responder (also known as suspect). The test reader animals were further tested using Comparative Intra-dermal Tuberculin test (CITT) in neck region to identify selectively the reactivity to bovine TB and para TB [7].

\subsection{Pathological investigation}

\subsubsection{Post mortem examination}

Using extremely safety precaution, tuberculin test positive dairy cattle $(\mathrm{N}=5)$ were sacrificed and necropsies at disposal areas of BAU Dairy Farm during a period between 2013 and 2014. The tuberculin test positive cattle were euthanized by intravenous injection of saturated $\mathrm{MgSO}_{4}$ solution and a thorough postmortem examination was carried out. Aseptically lungs, liver, kidney, spleen and lymphnode (prescapular, prefemoral, mediastinal and mesenteric) were investigated for the presence of tuberculous nodules. These organs were sliced into $2 \mathrm{~cm}$-thick to facilitate detection of tuberculous nodules. These organs were, therefore, collected for histopathological investigation and extraction of genomic DNA for PCR detection of specific causes of TB. The carcases with all of the excreta were buried in soil and protected from scavenging animals.

\subsubsection{Histopathological examination}

The lungs, liver, heart, spleen, lymph node and kidney were collected following necropsy, fixed in $10 \%$ buffered neutral formalin and stained with H\&E and Ziehl-Neelsen staining [8].The stained sections were examined at low (10x) and high power (100x) microscopic field. The images were captured and analyzed.

\subsection{PCR detection of Mycobacterium spp. \\ 2.3.1 Extraction of bacterial DNA}

Portion of lungs were collected aseptically, snap frozen and used for bacterial DNA extraction and detection of TB by using multiplex and uniplex PCR. In brief, $2 \mathrm{gm}$ frozen tissues were crushed in liquid nitrogen and extracted bacterial DNA using conventional method [9]. The quality and quantity of the extracted DNA was measured by using agarose gel electrophoresis and spectrophotometry (SpectronicR Genetics ${ }^{\mathrm{TM}} \mathrm{New}$ York, USA) (A260/A280).

\subsubsection{PCR amplifications of selected genomes}

The multiplex PCR was used to detect Mycobacterial infection in general (Table 1). The uniplex PCR was adapted and designed to differentiate infectivity due to M. bovis and M. tuberculosis. BCG vaccine strain and a known positive human case of TB due to $M$. tuberculosis were used in PCR as positive control for $M$. bovis and $M$. tuberculosis respectively. The known positive PCR reaction mixture containing BCG vaccine DNA and DNA from human TB due to M. tuberculosis without set primers were used in PCR as the negative control. Positive and negative controls were always included in the PCR settings to evaluate the degree of reactivity and specificity. The Promega ${ }^{\circledR}$ PCR kit, USA used was $2 x$ in strength and individual primer used per reaction was $20 \mathrm{pmol}$. A total of $25 \mu 1$ volume of PCR reaction mixture (Table 2 ) was always used in a Thermal cycler (Eppendorf, Germany) at controlled condition. 
The PCR was held at $-4^{\circ} \mathrm{C}$, the amplicons were analyzed by electrophoresis in $1.5 \%$ agarose gel containing ethidium bromide $(0.5 \mu \mathrm{g} / \mathrm{ml})$ and images were capture using an automated gel documentation system (Cell Biosciences, Alphalmager HP, USA).

\section{Results and Discussion}

Globally TB has been a major cause of morbidity and mortality of men and animals. The distinct test results of tuberculin, gross and microscopic pathology, virulence and host tropism could not differentiate infectivity due to $M$. bovis and $M$. tuberculosis at farm level. Due to the presence of saprophytic mycobacterium, high genome sequence homology among species of Mycobacterium and the lack of species-specific genes for each of the M. bovis and M. tuberculosis, it is suggested that distinctive protocols are needed to detect and differentiate these bacilli. The genomes of M. bovis and M. tuberculosis showed $99.95 \%$ identity and detection and differentiation of the etiology of TB is another hurdle. Even after the PCR amplification of selected genes may be of value to detect and differentiate infectivity due to M. bovis and M. tuberculosis if primers were designed from base deletion regions. Literature available indicated that the occurrence of bovine and human TB in cattle is common in many developing countries [10] and this may be due to lack of appropriate technology to detect TB at early onset. The traditional tests protocols such as intradermal tuberculin test, necropsy, histopathology, and Ziehl-Neelsen staining of smears and tissue section invariably may be used to understand pathobiology of TB in cattle. These technologies were used in this study and efficacies of these tests were evaluated on farms and in the laboratory.

\subsection{Tuberculin test}

The tuberculin test was performed on to the caudal fold of the tail of randomly selected dairy cattle [11]. Caudal fold is the highest sensitive area for performing tuberculin test [12] rather than skin of neck region. Intradermal tuberculin test is less frequently used in dairy cattle of Bangladesh to identify BTB. In this study, out of 100 dairy cattle tested using caudal fold tuberculin test, a total of $5(5 \%)$ appeared positive to bovine TB. Bovine PPD is used in farm animal is ideal reactor to both bovine and human TB[13], [14], [15]. The test positive animals were, therefore, tested with comparative intradermal tuberculin tests in neck region according to set criteria as proposed by [7]. While PPD was injected, the animal's immune system recognizes the PPD, inflammatory cells (white blood cells) migrate to the injection site to get rid of PPD. This response was recognized by swelling at the injection site [16], [17] that persisted for more than 48 hours. None of the cattle developed hypersensitive reaction at the injection sites due to Avian PPD. The test reactor animals were infected with either bovine TB or human TB.

\subsection{Clinico-pathological investigation of tuberculin test positive cattle \\ 3.2.1 Clinical observations}

The tuberculin test positive animals on clinical examination $(\mathrm{N}=5)$ showed enlargement of prescapular lymphnodes in two cases. However, insignificant difference of the body condition score (score 2 to 3) [18] was observed between tuberculin test positive and test negative cattle. On quarantine, two tuberculin test positive cattle showed progressive emaciation, reduce feed intake and recumbency (Figure 1a). Three tuberculin test positive cattle did not show any sign of illness even after six months of testing. These animals were again tested with bovine PPD and also gave positive results. On clinical observation $(\mathrm{N}=02)$ abnormalities in cattle revealed were respiratory distress, soft and chronic cough, the characteristics clinical signs and symptoms of TB in cattle [11], [19], [20]. One cattle (No. 3) did not show any sign of illness but were positive to tuberculin test. The infected and test positive cattle were sacrificed to prevent dissemination of infection in healthy cattle, susceptible animals and humans [21].

\subsection{Necropsy of Tuberculin test positive animal}

In order to reduce infectivity in farm animals due to TB it is recommended to test and slaughter of tuberculin test positive animals at early onset [7]. To avoid zoonosis, special extreme care was taken during necropsy. To ensure and differentiate infectivity in cattle due to M. tuberculosis and M. bovis, five tuberculin test positive cattle were slaughtered and necropsies. The fleshes, lungs, lymphnode, liver, kidney, spleen and bone marrow of three test positive cattle appeared paler. The characteristic tuberculous nodules were seen in the lungs of two cattle. The mandibular $(\mathrm{N}=01)$, prescapular $(\mathrm{N}=01$, Figure $1 \mathrm{~b})$, bronchial $(\mathrm{N}=02)$, mediastinal $(\mathrm{N}=01)$ and mesenteric ( $\mathrm{N}=02$, Figure 1c) lymphnodes appeared swollen. Three out of five tuberculin test positive cattle having generalized TB and one had TB localized to prescapular lymphnode.

Noticeable lesions were most frequent in lungs followed by mesenteric and bronchial lymph nodes. In lungs, calcification of lesions was not observed. However, infected animals have associated lesions like pipesteam liver $(\mathrm{N}=03)$, bottle jaw $(\mathrm{N}=01)$ and terminal recumbency $(\mathrm{N}=01)$. Previous investigators also reported similar findings at necropsy [11], [22], [23], [24]. In lungs miller's seed appearance (Pearl disease) is also 
Evaluate comparative efficacy of tuberculin test, gross and histopathology, Ziehl-Neelsen staining..

indicative for bovine TB [25] Pipe steam liver or bottle jaw due to fascioliasis could have predispose animal to clinical form of TB and could be a potential risk factor [21],[26]. Moreover, these kinds of lesions were more or less alike but meningitis [25] and TB-like lesions in the skin of cattle [19] were not observed in this investigation. [25] reported disseminated TB granulomas in the pleural and peritoneal surfaces of bovines. [27] noted pathological lesions in the ovaries in association with bilateral ovarian haematoma and tuberculosis. However, tuberculous meningitis, Pearl disease and myeloid form of TB were not seen in cattle.

\subsection{Histopathological examination}

The representative tissues stained with H\&E and Ziehl Neelsen staining, examined under a microscope showed granulomatous inflammations. The inflammatory infiltration was dominated with epithelioid cells, macrophages (Figure 2), lymphocytes, plasma cells, fibroblasts and caseous necrosis in the center of the lesion. Apart from lymphnode, lungs showed typical granulomatous reaction. Characteristic typical granulomas containing central core of caseous necrosis surrounded by mononuclear cells and fibroblast in the peribronchiolar and in lungs tissues. The lymphoid follicles of lymphnodes were lightly packed with lymphocytes, indicating depletion of lymphocytes (Figure 2c). The granulomatous nodules of enlarged and affected lymphnodes were consisting of central core of caseous necrosis and peripheral deposition of lymphocytes and macrophages. Previous authors also reported microscopic lesions in the lungs, lymphnodes and spleen of TB infected cattle [28], [29]. In earlier report, lesions in lungs, lumen of bronchioles, lymphnodes, spleen and liver were reported [23], [30], [31]. The intensities of lesions in lungs were reported to heavier than other organs. This is because of the bacteria enters into the cattle by inhalation [32]. Then begin to multiply in lungs and spread to the lymph nodes [33] Basically, TB bacilli infect alveolar macrophages which can transport the bacilli to local lymph nodes, and then spread through the bloodstream. Macrophages phagocytosed bacilli which subsequently interact with cells involved in innate and acquired immune responses in tissue or draining lymphnodes resulting nonvascular nodular granulomas known as 'tubercles'. The characteristic tuberculous lesions were frequently occur in lungs, retropharyngeal, bronchial, mediastinal, mesenteric lymph nodes, liver, spleen, serous membranes, pleura, uterus and or in mammary gland [19], [32], [34]. In this study, Ziehl-Neelsen staining of the impression smears of lungs and lymphnode revealed organisms as slender pink rods. The concentration of acid fast bacteria was higher in lungs lesion than lymphnodes. The acid fast bacilli were evident in tissue section by Ziehl-Neelsen staining [29], [35] but the bacilli was not detected in any tissue in cattle No. 3. However, demonstration of the organisms in tissue section, exudates or excretions can easily be made by using acid fast staining [25] but the species identification of Mycobacterium cannot be confirmed. However, molecular detection technique like PCR can be used to detect distinguishable gene of bovine or human TB [36] from clinical samples. This study used samples in PCR technique for specific detection of TB in cattle due to $M$. bovis and $M$. tuberculosis. In comparison with H\&E and acid fast staining, PCR technique appeared as more sensitive and accurate test protocol to detect TB in cattle [21], [37]. The multiplex PCR used (Figure 3a) selectively detected infectivity in cattle due to Mycobacterium genus (1030bp) and also due to MTB complex (372bp). Uniplex PCR was, therefore, used to differentiate infectivity due to M. tuberculosis from $M$. bovis with the DNA extracted from lungs of tuberculin test positive cattle. [38] designed a PCR targeting MPB gene of M. bovis (600bp), this PCR was used in this study. Out of five cattle tested in PCR targeting MPB83 gene, only three cattle found to generate $600 \mathrm{bp}$ amplicons and they were infected with M. bovis. To identify infectivity due to $M$. tuberculosis, a uniplex PCR was, therefore, designed targeting H37Rv3479HP gene (667bp). The primers Rv3479HPF and Rv3479HPR designed and used in PCR found to generate 667bp amplicon in a case (cattle No. 1). Out of 5 samples tested in PCR, three cattle (cattle No. 2,4 and 5) were infected with $M$. bovis and one cattle (No. 1) was infected with M. tuberculosis. Infectivity in cattle No. 3 due to either M. bovis or M. tuberculosis spp. could not identified by using necropsy, histopathology and PCR techniques. This cattle may have saprophytic Mycobacterium or myeloid form of $\mathrm{TB}$, required further investigation.

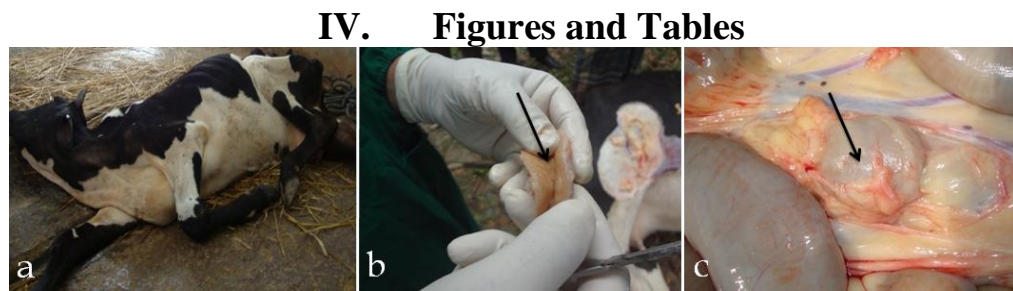

Figure 1: Investigation of tuberculin test positive cattle (a) at terminal recumbancy. The prescapular lymphnodes (b, black arrow) of a cow appeared larger and containing tuberculous nodules. The mesenteric lymph nodes (c, black arrow) also appeared larger in a tuberculin test positive cattle and containing caseous necrotic masses. 




Figure 2: Histopathologic investigation of the sections of lungs (a and b), spleen (c) and prefemoral lymphnode (d) of tuberculin test positive cattle stained with H\&E (10x). Typical granulomas (white circle) were seen in lungs, spleen and lymph node consisting of epitheloid cells, lymphocytes and fibrous connective tissues.

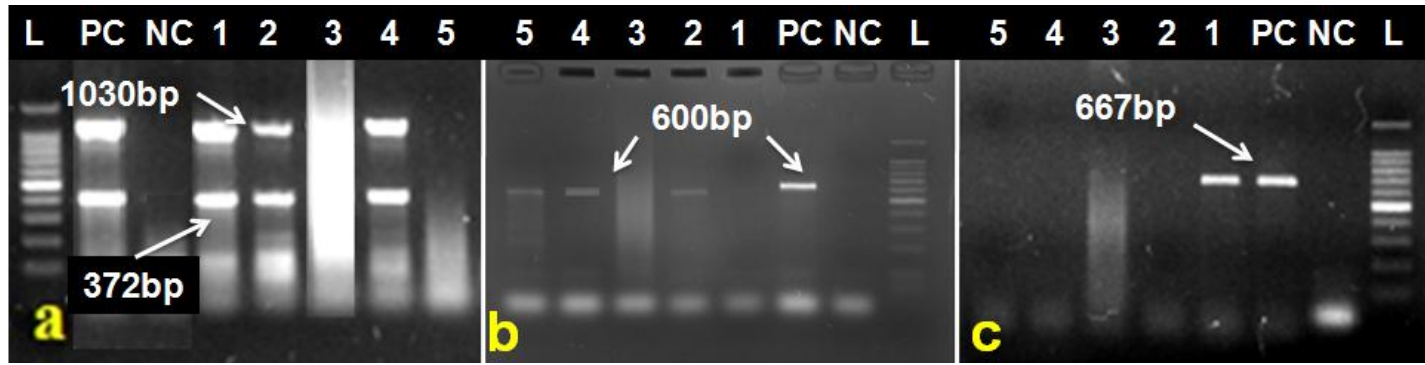

Figure 3: Multiplex (a) and uniplex (b and c) PCR detection of the causes of TB with the DNA extracted from the lungs of tuberculin test positive cattle. The genes targeted were 16srRNA (a), MPB83 (b) and H37Rv3479HP for the detection of Mycobacterium genus and MTB complex (a, 1030bp and 372bp), infection due to M. bovis $(600 \mathrm{bp}, \mathrm{b})$ and M. tuberculosis $(667 \mathrm{bp}, \mathrm{c})$. The lane $\mathrm{L}$ is for $100 \mathrm{bp}$ ladder, PC is for positive control, NC is for negative control and lane 1 to 5 were for test samples. Out of five cattle DNA tested in PCR, three were infected with M. bovis, one with M. tuberculosis. One cattle (Cattle N. 3) showed tuberculin test reactivity but did not have gross and microscopic lesions in the organs investigated and did not generate gene specific amplicons in PCR.

Table 1: Oligonucleotide primers used to amplify selected genomes of Mycobacterium in PCR. The multiplex PCR used to amplify fragments of 16sr RNA (1030bp, 180bp, 850bp and 372bp). The uniplex PCR used to amplify fragments of MPB83 (600bp) and H37Rv Rv3479HP genes for the detection of M. bovis and M. tuberculosis respectively.

\begin{tabular}{|c|c|c|c|}
\hline Primers name & Sequences (5'-3') & Product Size (bp) & References \\
\hline $\begin{array}{l}\text { MYCGEN-F1 } \\
\text { MYCGEN-R1 } \\
\text { MYCAV-R2 } \\
\text { MYCINT-F2 } \\
\text { TB 1-F3 } \\
\text { TB1-R3 }\end{array}$ & $\begin{array}{l}\text { agagtttgatcctggctcag } \\
\text { tgcacacaggccacaaggga } \\
\text { accagaagacatgcgtcttg } \\
\text { ccttaggcgcatgtctita } \\
\text { gaacaatccggagttgacaa } \\
\text { agcacgctgtcaatcatgta }\end{array}$ & $\begin{array}{l}1030 \text { (F1R1) Mycobacterium spp. } \\
180(\mathrm{~F} 1 \mathrm{R} 2) \text { M avium } \\
850(\mathrm{~F} 2 \mathrm{R} 1) \text { M. intracellulare } \\
\text { 372(F3R3) MTB complex }\end{array}$ & $\begin{array}{l}\text { Wilton and } \\
\text { Cousins, } 1992\end{array}$ \\
\hline MPB83F & cagggatccaccatgttcttagcgggttg & $600 \mathrm{M}$. bovis & Jiang et al., \\
\hline MPB83R & tggcgaattcttactgtgccggggg & & \\
\hline H37Rv3479HPF & gaactcaccgtcggtggtga & 667 M. tuberculosis & Designed \\
\hline H37Rv3479HPR & ccttgctcgatctctgcgtc & & \\
\hline
\end{tabular}

Table 2: Thermal profile used to amplify selected genomes of Mycobacterium in multiplex and uniplex PCR. The thermal profiles set up following a number of trial and error with the primers and genomic DNA in a thermal cycler

\begin{tabular}{|c|c|c|c|c|c|c|}
\hline \multirow[t]{3}{*}{ Primers pair used } & \multicolumn{6}{|c|}{ Thermal profile } \\
\hline & \multirow{2}{*}{$\begin{array}{l}\text { Initial } \\
\text { denaturation }\end{array}$} & \multicolumn{4}{|l|}{ PCR Cycles } & \multirow{2}{*}{$\begin{array}{l}\text { Final } \\
\text { Elongation }\end{array}$} \\
\hline & & Denaturation & Annealing & Elongation & No. of Cycles & \\
\hline $\begin{array}{l}\text { MYCGEN-F1 } \\
\text { MYCGEN-R1 } \\
\text { MYCAV-R2 } \\
\text { MYCINT-F2 } \\
\text { TB 1-F3 } \\
\text { TB1-R3 }\end{array}$ & $94^{0} \mathrm{C}, 5 \mathrm{~min}$ & $94^{0} \mathrm{C}, 30 \mathrm{sec}$ & $62^{\circ} \mathrm{C}, 2 \mathrm{~min}$ & $75^{\circ} \mathrm{C}, 2 \mathrm{~min}$ & 40 & $75^{\circ} \mathrm{C}, 7 \mathrm{~min}$ \\
\hline
\end{tabular}


Evaluate comparative efficacy of tuberculin test, gross and histopathology, Ziehl-Neelsen staining..

\begin{tabular}{|l|l|l|l|l|l|l|}
\hline $\begin{array}{l}\text { MPB83F } \\
\text { MPB83R1 }\end{array}$ & $98^{\circ} \mathrm{C}, 5 \min$ & $95^{\circ} \mathrm{C}, 1 \mathrm{~min}$ & $56^{\circ} \mathrm{C}, 1 \mathrm{~min}$ & $72^{\circ} \mathrm{C}, 1 \mathrm{~min}$ & 32 & $72^{\circ} \mathrm{C}, 10 \mathrm{~min}$ \\
\hline $\begin{array}{l}\text { H37Rv3479HPF } \\
\text { H37Rv3479HPR }\end{array}$ & $94^{\circ} \mathrm{C}, 5 \min$ & $94^{\circ} \mathrm{C}, 30 \mathrm{sec}$ & $57^{\circ} \mathrm{C}, 1.5 \mathrm{~min}$ & $72^{\circ} \mathrm{C}, 3 \min$ & 35 & $72^{\circ} \mathrm{C}, 5 \mathrm{~min}$ \\
\hline
\end{tabular}

\section{Conclusion}

This study adapted traditional and molecular test protocol to detect TB in cattle and differentiate infectivity due to M. bovis and M. tuberculosis. The tuberculin test protocol applied on 100 dairy cattle showed hypersensitive reactivity (bovine PPD) in five cattle. None of the cattle developed reactivity to avian PPD. At necropsy and histopathology granulomatous nodules were seen in four cases. The uniplex and multiplex PCR used successfully detected infectivity due to $M$. bovis and M. tuberculosis in four cases. In a case of tuberculin test positive cattle, infectivity due to Mycobacterium spp was not detected by using pathological investigation and PCR and these cattle may have localized TB which was unable to detect or may be infected with saprophytic Mycobacterium. The uniplex and multiplex PCR test protocols appeared sensitive and accurate detection tool to detect specific causes of TB in dairy cattle. M. bovis and M. tuberculosis are extremely zoonotic pathogens and present in the cattle of BAU dairy farm. People working in dairy farms and farm premises are at risk to the infectivity of TB; they need to sit for regular tuberculin and cough testing and identify the infected case at early onset. Mycobacterium derived from cattle is usually multidrug resistance, technologies need to adapt or design to detect drug resistance $\mathrm{TB}$ and prevent future casualty.

\section{Acknowledgements}

Thanks are due to Bangladesh Agricultural Research Council (SPGR, PIU-BARC, NATP Phase-1, Project Id No. 307) for funding the research.

[1]. BBS 2011. http://www.bbs.gov.bd/Home.aspx

\section{References}

[2]. C. Thoen, P. Lobue, I. de Kantor, The importance of Mycobacterium bovis as a zoonosis. Veterinary Microbiology,112(24),2006,339-345

[3]. U. Yumi, M. Tooru, Tuberculosis as a zoonosis from a veterinary perspective, Comparative Immunology, Microbiology and Infectious Diseases, 30, 2007, 415-425.

[4]. M. Raviglione, Global tuberculosis report, 20 $0^{\text {th }}$ edition. World Health Organization, 20 Avenue Appia, 1211 Geneva 27, 2015, Switzerland

[5]. L. Chen, J. Wang, A. Zganiacz, Z. Xing, Single Intranasal Mucosal Mycobacterium bovis BCG Vaccination Confers Improved Protection Compared to Subcutaneous Vaccination against Pulmonary Tuberculosis, Infection and Immunity,72(1),2004,238-246.

[6]. Kekkaku, Laboratory media for the cultivation of tubercle bacillus, Article in Japanese Review,73(5), 1998, 329-37.

[7]. OIE, Manual of Diagnostic Tests and Vaccines for Terrestrial Animals International Office of Epizootic (OIE). Assoc. Paris, 31, 2008, 213-225.

[8]. L.G. Luna, Manual of Histologic Staining Methods of the Armed Forces Institute of Pathology, (New York: McGraw Hill Book Co, 1968).

[9]. S.S. Labony, N. Begum, U.K. Rima, M.G.A. Chowdhury, M.Z. Hossain, M.A. Habib, and M.A.H.N.A. Khan, Apply traditional and molecular protocols for the detection of carrier state of visceral leishmaniasis in black Bengal goat, IOSR Journal of Agriculture and Veterinary Science (IOSR-JAVS),7(2),2014,13-18

[10]. M.A. Forrellad, L. Klepp, A. Gioffré, J.S.Y. García, H.R. Morbidoni, M. de la Paz Santangelo, A.A. Cataldi, and F. Bigi, Virulence factors of the Mycobacterium tuberculosis complex, Virulence, 4(1), 2013, 1-64.

[11]. OIE, Bovine Tuberculosis. An OIE Collaborating Center Iowa State University College of Veterinary Medicine, USA. www.cfsph.iastate.edu/II CAB, 2009, 1-6.

[12]. N.K. Isabel, A.C. Odeon, Steffan, M.J. Auza, C.R. Madrid, N. Marchevsky, Sensitivity of the cervical and the caudal fold tuberculin tests with Mycobacterium bovis in infected cattle of Argentina, Revue scientifique et technique Del Office International Des Epizootics, 3(1),1984,137-150.

[13]. G. Ameni, A. Aseffa, H. Engers, D. Young, S. Gordon, G. Hewinson, and M Vordermeier, High prevalence and increased severity of pathology of bovine tuberculosis in Holsteins compared to zebu breeds under field cattle husbandry in central Ethiopia, Clinical and Vaccine Immunology, 14(10),2007,1356-1361.

[14]. M.M. Islam, M.A.R. Siddique, M.A. Haque, M.A. Baki, S. Majumder, J.J. Parrish, and M. Shamsuddin, Screening some major communicable diseases of AI bulls in Bangladesh, Livestock Research Rural Development, 19,2007,79.

[15]. B.J. Trangadia, S.K. Rana, and V.A. Srinivasan, Prevalence of bovine tuberculosis in organized dairy farm, Indian journal of veterinary pathology, 37(1), 2013, 72-74.

[16]. H.J. Pharo, A. Motalib, S.F. Routledge, and S. Alam, The prevalence of bovine tuberculosis in the Bangladesh Cattle Development Project, Bangladesh Veterinary Journal, 15, 1981, 53-56.

[17]. M.M. Rahman, and M.A. Samad, Prevalence Of Bovine Tuberculosis And Its Effects On Milk Production In Red Chittagong Cattle, Bangladesh Journal of Veterinary Medicine, 6(2), 2008, 175-178.

[18]. Rodenburg J, Body Condition Scoring of Dairy Cattle, Ontario Ministry of Agriculture and Food, Queen Printers for Ontario, www.omafra.gov.on.ca, 2013.

[19]. O.M. Radostits, C.C. Gay, D.C. Blood, and K.W. Hinchdiff (Ed.), Disease caused by mycobacterium spp.--IV. In: Veterinary Medicine (A text book of the diseases of cattle, sheep, pigs, goats and horses. 9th edition. W.B. Saunders Company Ltd, London, 2000, 909-917

[20]. C.M. Fraser, H.E. Amstutz, and J. Archibald (Ed.), Tuberculosis (In: the Merck veterinary Manual. Sixth edition. Published by Merck \& CO., Inc. Rahway, N.J., USA, 1986. 
Evaluate comparative efficacy of tuberculin test, gross and histopathology, Ziehl-Neelsen staining..

[21]. M.Z. Hossain, The incidence, pathology, diagnosis and molecular characterization of bovine tuberculosis in Bangladesh, doctoral diss., Department of Pathology, Bangladesh Agricultural University, Mymensingh-2202, Bangladesh, 2015.

[22]. S.I. Cadmus, N.N. Atsanda, S.O. Oni, and E.E.U. Akang, Bovine tuberculosis in one cattle herd in Ibadan in Nigeria, VeterinarniMedicina, 49(11), 2004,406-412.

[23]. S.A. Hena, B.K. Tanimomo, R. Tarhyel, E.O. Ngbede, O.O. Oluwasina, G.P. Jagtap, M.C. Dhavale, and U. Dey, A tentative case of tuberculosis detected at postmortem examination of animal carcasses slaughtered at some local slaughter slabs in north-western Nigeria, Scientific Journal of Agricultural,1(2),2012,27-32.

[24]. A. Pockevicius, P. Maciulskis, and K. Lukauskas, Immunopathomorphology of bovine tuberculosis. Veterinarija-ir-Zootechnika,22, 2003,15-21.

[25]. T.C. Jones, R.D. Hunt, and N.W. King (6th Ed.), Veterinary Pathology, (USA: Wiley, John \& Sons, Incorporated, 1997) 489-497.

[26]. R.J. Flynn, C. Mannion, O. Golden, O. Hacariz, and G. Mulcahy, Experimental Fasciola hepatica Infection Alters Responses to Tests Used for Diagnosis of Bovine Tuberculosis, Infection and Immunity,75 (3), 2007, 373-1381

[27]. M. Fathalla, N. Hailat, S.Q. Lafi, E.A. Basha, and A. Al-Sahli, An abattoir survey of gross reproductive abnormalities in the bovine genital tract in Northern Jordan, Israel Journal of Veterinary-Medicine,55(3), 2000, 83-87.

[28]. Q. Nahar, M. Pervin, M.T. Islam, and M.A.H.N.A Khan, Application of PCR for the detection of bovine tuberculosis in cattle, Journal of Bangladesh Agricultural University, 9(1), 2011,73-78.

[29]. Z. Rahim, M. Mollers, K.V. Arianne, B. Jessica, K. Zaman, M.A. Matin, M. Kamal, R. Raquib, S. Dick van, M.A. Baqi, G.C. Frank Heilmann and G.M. Adri, Characterization of Mycobacterium africum subtype I amoung cows in a dairy farm in Bangladesh using spoligotyping, Southeast Asian Journal of Tropical Medicine and Public Health, 38, 2007,706-713.

[30]. J.E. Shitaye, B. Getahun, T. Alemayehu, Skoric, F. Treml, P. Fictum, V. Vrbas, and I. Pavlik, A prevalence study of bovine tuberculosis by using abattoir meat inspection and tuberculin skin testing data, histopathological and IS6110 PCR examination of tissues with tuberculous lesions in cattle in Ethiopia, Veterinarni Medicina,51(11),2006,512-522.

[31]. A.A. Mosaad, A.S. Abdel-Hamed, S.I. Fathalla, A.A. Ghazy, S. Elballal, A. Elbagory, H. Mahboub, K. Gaafar, K.E. Elgayar, A.S. Mohamed, A.I. Amin, M.A. Akeila, and H.A. Abdelrahman, Sensitive and Specific Diagnostic Assay for Detection of Tuberculosis in Cattle, Global Veterinaria,8(6),2012,555-564.

[32]. S.D. Neill, J.M. Pollock, D.B. Bryson, and J. Hanna, Pathogenesis of Mycobacterium bovis infection in cattle, Veterinary Microbiology, 40,1994, 41-52

[33]. M. Numata, M. Nishikawa, M. Kudo, T. Kaneko, H. Ikeda, T. Okubo, Y. Rino, and H. Kitamura Pulmonary tuberculosis with systemic lymph node swelling, Nihon Kyobu Shikkan Gakkai Zasshi,33(11),1995,1270-1275.

[34]. M. Minakuchi, E. Tanaka, T. Inoue, M. Sakuramoto, Y. Maeda, K. Maniwa, and Taguchi Y, An adult case of cervico-mediastinal lymph nodes tuberculosis followed by the development of pulmonary lesions during the treatment with antituberculous drugs, Kekkaku, 80(1), 2005, 19-23.

[35]. A.H. Selwyn, Systemic bovine tuberculosis, a case report. Semina: Ciências Agrárias, Londrina, 23(1), 2002, 75-79.

[36]. P.A. Cristina, Q.F.L. Clarice, A.P. Karina, S.G.J. Klaudia, L.A.R.O. Ana, Mycobacterium bovis identification by a molecular method from post-mortem inspected cattle obtained in abattoirs of Mato Grosso do Sul, Brazil, Memorias Do Instituto Oswaldo Cruz, Rio de Janeiro, 100(7), 2005,749-752.

[37]. E.R. Rosa, L.G. Daniel, A.M. Gloria, M. William, E.P. Manuel, and A.M. Luis, Identification of Mycobacterium bovis in Bovine Clinical Samples by PCR Species-Specific Primers, Canadian Journal of Veterinary Research, 63(2),1999, 101-106.

[38]. H.G. Wiker, MPB70 and MPB83 - Major Antigens of Mycobacterium bovis. Journal of Immunology, 69, 2009, $492-499$. 